\title{
Modified Nonlinear Inverse Synthesis for Optical Links with Distributed Raman Amplification
}

\author{
S. T. Le ${ }^{1 *}$, J. E. Prilepsky ${ }^{1}$, M. Kamalian ${ }^{1}$, P. Rosa ${ }^{2}$, M. Tan ${ }^{1}$, J. D. Ania-Castanon ${ }^{2}$, P. Harper ${ }^{1}$ and S. \\ K. Turitsyn ${ }^{1}$ \\ ${ }^{1}$ Aston Institute of Photonics Technologies, Aston University, Birmingham, B4 7ET, UK, \\ ${ }^{2}$ Instituto de Optica, IO-CSIC, CSIC, Madrid, 28006, Spain \\ ”let1@aston.ac.uk
}

\begin{abstract}
We propose a modification of the nonlinear digital signal processing technique based on the nonlinear inverse synthesis for the systems with distributed Raman amplification. The proposed pathaverage approach offers $3 d B$ performance gain, regardless of the signal power profile.
\end{abstract}

\section{Introduction}

Nonlinear Fourier transform (NFT) and eigenvalue communication [1] with the use of nonlinear signal spectrum, both discrete [1-3] and continuous [4-6], have been recently discussed as a promising transmission method to combat the fibre nonlinearity impairments. The NFT-based transmission systems explore the fact that the dynamics of individual NFT modes (nonlinear spectrum modes) is essentially linear. As a result, the nonlinearityinduced cross-talk between them is effectively absent [1]. It was shown in [5, 6] that information can be directly encoded onto the continuous part of the nonlinear spectrum by using the nonlinear inverse synthesis (NIS) scheme. This technique can be effectively integrated with any modulation format, offering up to $4.5 \mathrm{~dB}$ performance gain, which is comparable with multi-step per span digital back propagation [5]. However, all transmission schemes employing the NFTs are based on the integrability of the underlying nonlinear Schrödinger equation (NLSE) [1]. As a result, formally, the NFT technique can be directly applied only to optical links with ultra-long fibre laser-based distributed Raman amplification that provides a flat quasilossless gain profile [7].

In this paper, for the first time we investigate the impact of a non-ideal Raman gain profile on the performance of NIS-based systems. We introduce a path-averaged NIS scheme to improve the system performance by $3 \mathrm{~dB}$. Without loss of generality, we consider here open-cavity random distributed feedback (DFB) laser-based amplification, as this scheme provides the best performance among various other Raman amplification schemes [8].

\section{Random DFB laser Raman amplifier}

The schematic design of the random DFB laser Raman amplifier that allows achieving $2^{\text {nd }}$ order pumping with a single wavelength pump is shown in Fig. 1(a). In this scheme, a high
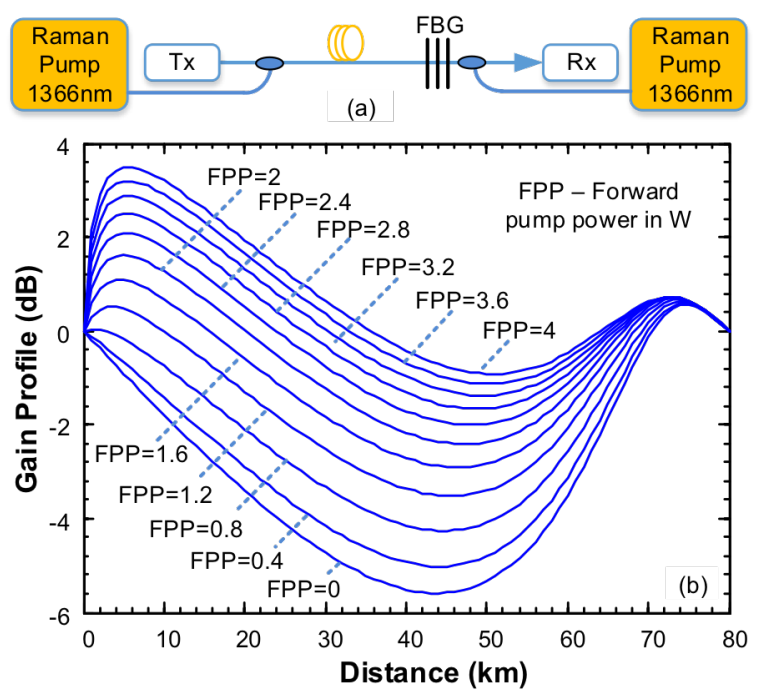

Fig. 1(a) schematic of random DFB laser Raman amplifier and (b) - gain profiles for different value of the forward pump power (FPP) and a span length of $80 \mathrm{~km}$.

reflectivity (99\%) fibre Bragg grating (FBG) centered at $1455 \mathrm{~nm}$ with $200 \mathrm{GHz}$ bandwidth is deployed at the end of the transmission span to reflect back-scattered Rayleigh Stokes-shifted light from the backward pump (at $1366 \mathrm{~nm}$ ) and create a random DFB laser at the frequency specified by the wavelength of the FBG. This random DFB laser acts as the first order pump that amplifies the signal at $1555 \mathrm{~nm}$. The lack of an FBG on the side of the forward pump significantly reduces the relative-intensity-noise transfer from the forward pump to the Stokesshifted light at $1455 \mathrm{~nm}$, which can seriously hinder coherent transmission [8].

Raman gain profiles for an $80 \mathrm{~km}$ length span are shown in Fig. 1(b) for different forward pump powers (FPP). The backward pump powers were chosen accordingly to provide a net gain of $0 \mathrm{~dB}$. We define the average gain variation (AGV) of the Raman gain profile as:

$A G V=\int_{z=0}^{L}(G(z)-k) d z /\left(L G_{0}\right), k=\int_{z=0}^{L} G(z) d z / L,(1)$

where $G(z)$ is the Raman gain, $L$ is the span length. The AGV is shown in Fig. 2 as a function 


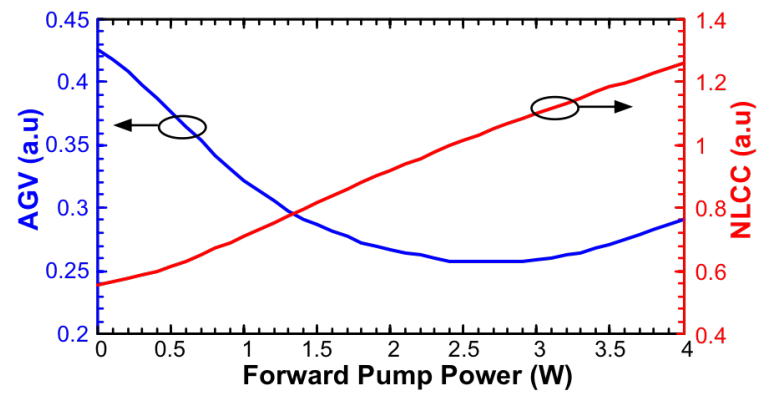

Fig. 2. AGV and NLCC as a function of the forward pump power; the span length was $80 \mathrm{~km}$.

of the FPP. The FPP $=0$ case corresponds to the backward-pumping-only scheme. From Fig. 2 we see that the AGV can be effectively reduced by increasing the FPP at the cost of increasing the system power consumption. The optimum value of FPP was found to be $\sim 2.7 \mathrm{~W}$, giving the $A G V \approx 0.255$.

\section{Modified NIS for non-ideal distributed Raman amplification}

The propagation of a complex slow-varying optical-field envelope $q(z, t)$ along a single-mode optical fibre is described by the NLSE [9]:

$$
j q_{z}-\beta_{2} q / 2+\gamma q|q|^{2}=j g(z) q
$$

where $t$ is the time in the frame co-moving with the group velocity of the envelope, $\beta_{2}<0$ is the dispersion coefficient, $\mathrm{Y}$ is the Kerr nonlinearity coefficient and $g(z)$ is the distributed gain/loss.

By introducing the standard change of variables

$$
q(z, t)=\exp \left(\int_{0}^{z} g(y) d y\right) A(z, t) \text {, Eq. (2) can be }
$$

rewritten as:

$$
j A_{z}-\beta_{2} A / 2+\gamma G(z) A|A|^{2}=0,
$$

which is the lossless NLSE with a distancedependent nonlinear coefficient; here $G(z)$ is the instantaneous gain, $G(z)=\exp \left(2 \int_{0}^{z} g(y) d y\right)$. We assume here that the dynamic of the envelope $A(z, t)$ does not change significantly after each fibre span. In this case, the distance-dependent nonlinear coefficient in (4) can be replaced by its averaged value over each fibre span, giving a lossless path-averaged (LPA) NLSE [9]:

$$
j A_{z}-\beta_{2} A / 2+\gamma\left(\int_{0}^{L} G(z) d z / L\right) A|A|^{2}=0
$$

Based on the LPA NLSE, we propose here the NIS scheme accounting for the non-ideal Raman gain profile as shown in Fig. 3(a). Firstly, the encoded complex signal $q(\mathrm{t})$ is normalized as follow:

$$
t / T_{s} \rightarrow t, z / Z_{s} \rightarrow z, q \sqrt{\gamma \cdot k \cdot Z_{s}} \rightarrow q
$$

where the time normalization $T_{s}$ is a free parameter (e.g., a characteristic time scale) and the associated space scale is $Z_{s}=T_{s}^{2} /\left|\beta_{2}\right| ; k$ is the nonlinear correction coefficient (NLCC) defined as in Eq. (1). The dependence of the NLCC on FPP is shown in Fig. 2 (red curve).

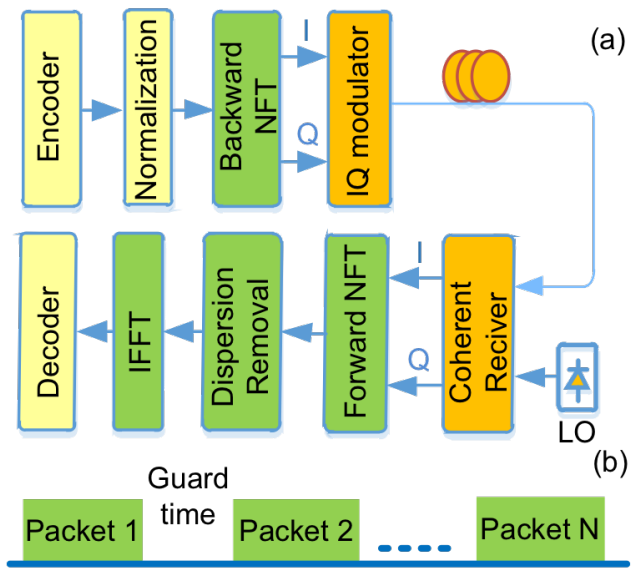

Fig. 3(a) - Block diagram of NIS-based transmission systems, (b) - Illustration of a burst mode transmission

After normalization, the linear Fourier spectrum of the encoded input waveform $(Q(\omega))$ is mapped onto the continuous part of the nonlinear spectrum using the inverse NFT. This defines the pre-processing of the initial signal at the transmitter and can be considered as nonlinearity pre-compensation. At the receiver, the nonlinear spectrum of the signal is retrieved using the forward NFT. Finally, single-tap dispersion compensation is performed to remove all deterministic impairments [5]. The details and numerical algorithms for the NFT and NIS techniques can be found in [5].

\section{Simulation setup and results and discussion}

To demonstrate how the proposed scheme works, we consider 16QAM 56-Gbaud OFDM NIS-based systems in the burst mode regime (Fig. 3(b)), as the NFT operations have to be performed on return-to-zero signals. In this scheme, the neighbouring packets are separated by a guard time, which is $20 \%$ longer than the dispersion induced memory. For simplicity, we assume that each packet data contains only one OFDM symbol. To generate the OFDM signals, the IFFT size of 1024 was used, where 112 subcarriers were filled with data (with Gray-coding) while the remaining subcarriers were set to zero for oversampling purpose. The propagation of signal in fibre link was simulated using the split-step Fourier method with a step size of $1 \mathrm{~km}$, using the gain profile shown in Fig. 1(a). The Raman noise was modelled as a Gaussian noise, which was added to the signal after each step $(1 \mathrm{~km})$, following the simulated noise profiles obtained according to [8]. The system performance was evaluated through the EVM and the estimated BER was converted to the $\mathrm{Q}$-factor.

The performance of OFDM systems with and without NIS, and with the proposed modified NIS scheme is compared in Fig. 4(a) for FPP $=0 \mathrm{~W}$ and $2.7 \mathrm{~W}$. It should be noted that by increasing the FPP, the Raman noise figure is reduced, which can be referred from the performance at 


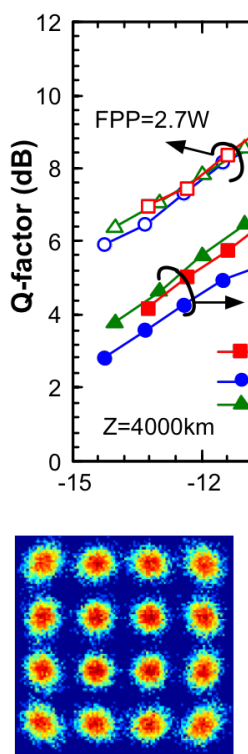

(b)
Fig. 4(a). Performance comparison of OFDM systems with and without NIS, and the modified NIS scheme, for different FPP values, (b)-(d) constellations at the optimum launch powers for system without NIS (b), with NIS (c) and with the modified NIS (d) schemes at FPP $=0 \mathrm{~W}$, respectively.

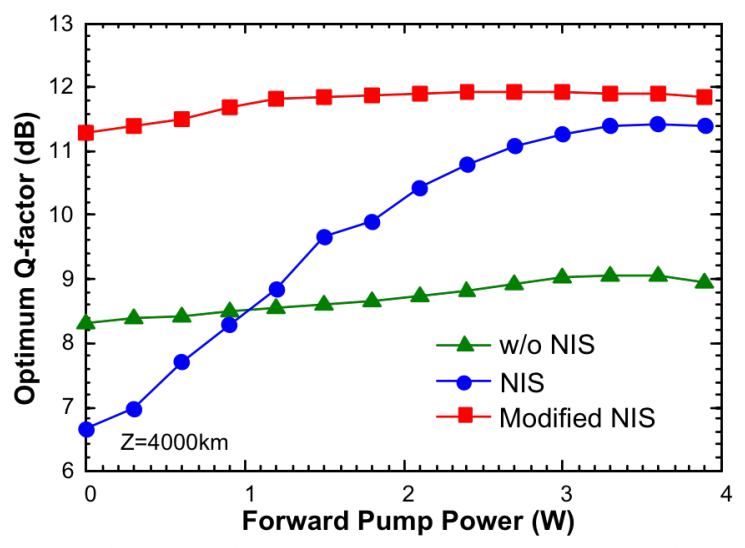

Fig. 5. Optimum Q-factor as a function of FPP for OFDM systems with and without NIS, and the modified NIS scheme. The transmission distance was $4000 \mathrm{~km}$

low signal powers. In Fig. 4(a) we see that the NIS method gives around $2 \mathrm{~dB}$ performance gain if $\mathrm{FPP}=2.7 \mathrm{~W}$. In this case, the $A G V$ is relatively small $(0.255)$, and the NIS scheme still brings about a meaningful performance gain. In addition, if one uses the modified NIS method based on eq. (4), an extra $1 \mathrm{~dB}$ gain can be achieved. However, when FPP $=0 \mathrm{~W}$, the direct use of the NIS method decreases the Q-factor by $\sim 2 \mathrm{~dB}$. This clearly indicates that the nonideal Raman gain profile has a significant impact on the NIS-based systems: When the AGV is high (AGV 0.43 if FPP=0W), the NIS method cannot produce any advantage due to the wrong power estimate. However, if the modified NIS scheme is employed, a Q-factor improvement of $\sim 3 \mathrm{~dB}$ is observed. This effectively means that the performance of the NIS scheme is enhanced by $5 \mathrm{~dB}$ by simply employing the NLCC that takes into account the non-ideal Raman gain profile. The received constellations at optimum launch powers for three systems under investigation are shown in Figs. 4(b)-(d).

The optimum $Q$-factors in systems with and without NIS and the modified NIS schemes are presented in Fig. 5 as functions of the FPP. As expected, when the FPP is increased from $0 \mathrm{~W}$ to $2.7 \mathrm{~W}$, the achievable performance of NISbased system increases dramatically as a result of the decrease in AGV. However, increasing further FPP, which increases the AGV accordingly, does not decrease the performance of NIS-based systems. We attribute this phenomenon to the reduction of Raman noise figure when the FPP is increased. In the other hand, the modified NIS scheme offers around $3 \mathrm{~dB}$ gain in Q-factor, independently of the FPP. As a result, if the modified NIS method is applied, forward pump is not necessary, offering a significant reduction in the cost and power consumption.

\section{Conclusion}

We have shown that the non-ideal Raman gain has a significant impact on the performance of NIS-based systems and proposed a modified NIS scheme offering a performance gain of $3 \mathrm{~dB}$, independently of the Raman gain profile.

\section{Acknowledgements}

This work was supported by UK EPSRC Grant UNLOC (EP/J017582/1) and H2020 Grant CHAOS (658982).

\section{References}

[1] A. Hasegawa and T. Nyu, "Eigenvalue communication," J. Lightwave Technol. Vol. 11, pp. 395-399 (1993).

[2] M. I. Yousefi et al, "Information transmission using the nonlinear Fourier transform, Parts I-III," IEEE Trans. Inf. Theory, Vol. 60, pp. 4312-4369 (2014).

[3] H. Bülow, "Nonlinear Fourier Transform Based Coherent Detection Scheme for Discrete Spectrum," OFC, paper We.3.K.2 (2015)

[4] E. G. Turitsyna and S. K. Turitsyn, "Digital signal processing based on inverse scattering transform," Opt. Lett. Vol. 38, pp. 4186-4188 (2013)

[5] S. T. Le, J. E. Prilepsky and S. K. Turitsyn, "Nonlinear inverse synthesis for high spectral efficiency transmission in optical fibers," Opt. Express. Vol. 22, pp. 26720-26741 (2014)

[6] S. T. Le, J. E. Prilepsky and S. K. Turitsyn, "Nonlinear inverse synthesis technique for optical links with lumped amplification," Opt. Express. Vol. 23, pp. 8317-8328 (2015)

[7] J-D. Ania-Castanon et. al., "Simultaneous Spatial and Spectral Transparency in Ultralong Fibre Lasers," Phys. Rev. Lett. 101, 123903 (2008)

[8] M. Tan et al "Long-haul Transmission Performance Evaluation of Ultra-long Raman Fibre Laser Based Amplification Influenced by Second Order Co-pumping," APC, paper ATh1E (2014)

[9] S. K. Turitsyn, B. G. Bale and M. P. Fedoruk, "Dispersion Management Soliton in fibre systems and laser", Phys. Rep. Vol. 521, pp. 135203(2012) 